\title{
Polarization-induced renormalization of molecular levels at metallic and semiconducting surfaces
}

\author{
García Lastra, Juan Maria; Rostgaard, Carsten; Rubio, A.; Thygesen, Kristian Sommer
}

Published in:

Physical Review B Condensed Matter

Link to article, DOI:

10.1103/PhysRevB.80.245427

Publication date:

2009

Document Version

Publisher's PDF, also known as Version of record

Link back to DTU Orbit

Citation (APA):

García Lastra, J. M., Rostgaard, C., Rubio, A., \& Thygesen, K. S. (2009). Polarization-induced renormalization of molecular levels at metallic and semiconducting surfaces. Physical Review B Condensed Matter, 80(24), 245427. https://doi.org/10.1103/PhysRevB.80.245427

\section{General rights}

Copyright and moral rights for the publications made accessible in the public portal are retained by the authors and/or other copyright owners and it is a condition of accessing publications that users recognise and abide by the legal requirements associated with these rights.

- Users may download and print one copy of any publication from the public portal for the purpose of private study or research.

- You may not further distribute the material or use it for any profit-making activity or commercial gain

- You may freely distribute the URL identifying the publication in the public portal 


\title{
Polarization-induced renormalization of molecular levels at metallic and semiconducting surfaces
}

\author{
J. M. Garcia-Lastra, ${ }^{1,2}$ C. Rostgaard, ${ }^{1}$ A. Rubio, ${ }^{2}$ and K. S. Thygesen ${ }^{1}$ \\ ${ }^{1}$ Center for Atomic-Scale Materials Design (CAMD), Department of Physics, Technical University of Denmark, \\ DK-2800 Kongens Lyngby, Denmark \\ ${ }^{2}$ ETSF Scientific Development Centre, Depto. Física de Materiales, Centro de Física de Materiales CSIC-UPV/EHU \\ and DIPC, Universidad del País Vasco (UPV)/EHU, Avenue Tolosa 72, 20018 San Sebastián, Spain \\ (Received 28 October 2009; published 21 December 2009; publisher error corrected 6 January 2010)
}

\begin{abstract}
On the basis of first-principles $G_{0} W_{0}$ calculations we systematically study how the electronic levels of a benzene molecule are renormalized by substrate polarization when physisorbed on different metallic and semiconducting surfaces. The polarization-induced reduction in the energy gap between occupied and unoccupied molecular levels is found to scale with the substrate density of states at the Fermi level (for metals) and substrate band gap (for semiconductors). These conclusions are further supported by self-consistent $G W$ calculations on simple lattice models. By expressing the electron self-energy in terms of the substrate's joint density of states we relate the level shift to the surface electronic structure, thus providing a microscopic explanation of the trends in the $G W$ and $G_{0} W_{0}$ calculations. While image charge effects are not captured by semilocal and hybrid exchange-correlation functionals, we find that error cancellations lead to remarkably good agreement between the $G_{0} W_{0}$ and Kohn-Sham energies for the occupied orbitals of the adsorbed molecule.
\end{abstract}

DOI: 10.1103/PhysRevB.80.245427 PACS number(s): 85.65.+h, 31.70.Dk, 71.10. $-\mathrm{w}, 73.20 .-\mathrm{r}$

\section{INTRODUCTION}

Solid-molecule interfaces are central to a number of important areas of physics and chemistry including heterogeneous catalysis, electrochemistry, molecular- and organic electronics, and scanning tunneling spectroscopy. ${ }^{1-4}$ Most of our current understanding of level alignment at interfaces builds on effective single-particle descriptions such as the Kohn-Sham scheme of density-functional theory (DFT) ${ }^{5}$ Within such theories the energy levels of a molecule close to a surface are determined by hybridization, charge-transfer, and interface dipole fields-all properties of the static mean field potential defining the single-particle Hamiltonian. On the other hand, from photoemission and electron transport measurements it is well known that the dynamic polarizability of the molecule's local environment can have a large influence on the level positions. ${ }^{6-10}$ Such polarization effects, which are induced by changes in the charge state of the molecule, are not captured by available single-particle descriptions.

Many-body perturbation theory provides a systematic method to obtain the true single-particle excitations [sometimes referred to as addition/removal energies or quasiparticle $(\mathrm{QP})$ energies] from the Green function of the system. In the $G_{0} W_{0}$ approximation the electron self-energy is written as a product of the (noninteracting) Green function and a dynamically screened Coulomb interaction, $\Sigma=i G_{0} W_{0} \cdot{ }^{11,12} \mathrm{It}$ is instructive to compare this to the bare exchange selfenergy given by $\Sigma_{x}=i G_{0} V$, where $V$ is the unscreened Coulomb interaction. It is well known that the Hartree-Fock (HF) eigenvalues correspond to energy differences between the $N$-particle groundstate and the unrelaxed $N \pm 1$-particle Slater determinants (Koopmans' theorem). The effect of replacing $V$ with the screened and frequency dependent $W_{0}$ is twofold: it introduces correlations into the many-body eigenstates, and it includes the response of the other electrons to the added electron/hole, i.e., relaxation effects. For a mol- ecule at a surface, the latter effect is particularly important as it incorporates the attractive interaction between the added electron/hole and its induced image charge, into the QP spectrum.

Recent experiments on molecular charge transport have renewed the interest for theoretical modeling of polarizationinduced level renormalization. First-principles $G_{0} W_{0}$ calculations for a benzene molecule on graphite ${ }^{13}$ as well as $\mathrm{CO}$ on $\mathrm{NaCl} / \mathrm{Ge}(001)^{14}$ have demonstrated significant reductions of

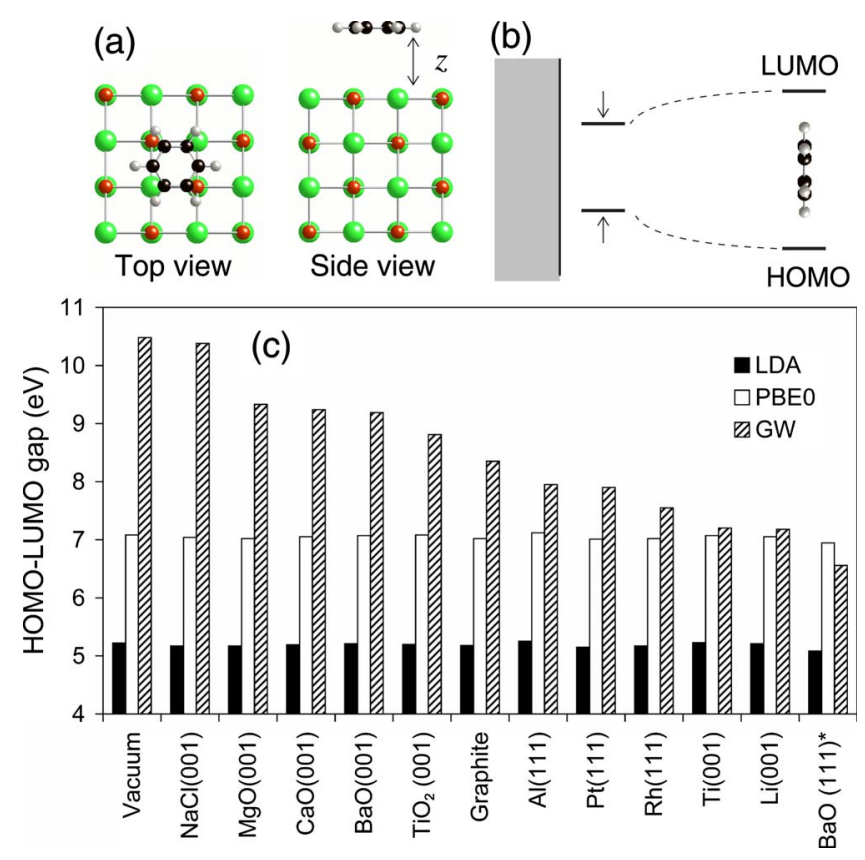

FIG. 1. (Color online) (a) Supercell used to represent benzene physisorbed on $\mathrm{NaCl}(001)$. (b) Reduction in a molecule's energy gap when it approaches a polarizable surface. (c) Calculated LDA, PBE0, and $G_{0} W_{0}$ HOMO-LUMO gap of a benzene molecule lying flat at $z=4.5 \AA$ above different surfaces. Note that $\mathrm{BaO}(111)$ is metallic due to surface states in the $\mathrm{BaO}$ band gap. 
the molecular energy gap due to image charge effects. Model $G W$ calculations have been used to elucidate the qualitative features of the effect across different bonding regimes. ${ }^{15}$ Classical electrostatic models of various complexities have been developed to correct energy levels obtained from single-particle calculations. ${ }^{16-19}$

In this work, we present a systematic study of image charge-induced renormalization at a range of different surfaces taking both a classical and quantum many-body viewpoint. We have performed DFT calculations with local density approximation (LDA) and hybrid (PBE0) exchangecorrelation functionals as well as $G_{0} W_{0}$ calculations for a benzene molecule weakly physisorbed on the metals $\mathrm{Li}, \mathrm{Al}$, $\mathrm{Ti}, \mathrm{Rh}, \mathrm{Pt}$, and the semiconductors/insulators $\mathrm{TiO}_{2}, \mathrm{BaO}$, $\mathrm{MgO}, \mathrm{CaO}$, and $\mathrm{NaCl}$. The results for the highest occupied molecular orbital (HOMO)- lowest unoccupied molecular orbital (LUMO) gap of benzene are shown in Fig. 1. While LDA and PBE0 yields a substrate independent HOMOLUMO gap, the $G_{0} W_{0}$ gaps are reduced from the gas phase value by an amount which depends on the polarizability of the surface. For all systems, we find that the dependence of the QP gap on the distance to the surface can be described by a classical image charge model. However, the model parameters are sensitive to the microscopic details of the system and this limits the usefulness of the classical model in pratice. By evaluating the $G_{0} W_{0}$ self-energy to second order we obtain a simple analytic expression which relates the level shift to the substrate's joint density of states weighted by Coulomb interaction matrix elements. The model suggests that the HOMO-LUMO gap should scale with the substrate band gap (for semiconducting surfaces) and density of states at the Fermi level (for metallic surfaces). This trend is verified for the first-principles results and is further supported by $G W$ calculations for simple lattice models. Finally, we analyze the deviation between the DFT and $G_{0} W_{0}$ results in more detail. We find that the occupied Kohn-Sham levels obtained with LDA (PBE0) are in very good agreement with the $G_{0} W_{0}$ results for benzene adsorbed on the metallic (semiconducting) surfaces, and we show that this is a result of significant error cancellation in the LDA/PBE0 approximations.

The paper is organized as follows. In Sec. II we outline the methodology used for the first-principles and model $G W$ calculations. In Sec. III we investigate to what extent the first-principles $G_{0} W_{0}$ results can be explained by a classical image charge model. In Sec. IV we derive a simple analytical expression for the polarization-induced level shift and show that it explains the main trends in both the first-principles as well as the model calculations. At the end of the section we analyze the description of occupied and unoccupied levels separately and discuss the effect of error cancellations in the DFT results. We conclude in Sec. V

\section{METHODS}

\section{A. $A b$ initio $G_{0} W_{0}$ calculations}

To model the solid-molecule interfaces we use a slab containing four atomic layers of the substrate in the experimentally most stable phase and a benzene molecule lying flat above the surface followed by $12 \AA$ of vacuum. The benzene molecule is not relaxed on the surface but is fixed in its gas phase structure at a distance $z$ from the surface. An example of a supercell is shown in Fig. 1(a) for the case of benzene on $\mathrm{NaCl}(001)$. The number of atoms included in the supercell per atomic layer is 9 for $\mathrm{Al}, \mathrm{Rh}, \mathrm{Pt}, \mathrm{Ti} ; 12$ for $\mathrm{Li}$ and $\mathrm{TiO}_{2}$; and 16 for $\mathrm{NaCl}, \mathrm{MgO}, \mathrm{CaO}$ and $\mathrm{BaO}$. This corresponds to distances between periodically repeated benzene molecules in the range 8.1 to $9.9 \AA$. All DFT calculations have been performed with the PWSCF code $^{20}$ which using norm-conserving pseudopotentials. $^{21}$ For exchangecorrelation functionals we have used the local density approximation $^{22}$ as well as the PBE0 hybrid functional. ${ }^{23,24}$ The Brillouin zone (BZ) was sampled on a $4 \times 4 \times 1 k$-point mesh, and the wave functions were expanded with a cut-off energy of 40 Hartree.

In the $G_{0} W_{0}(\mathrm{LDA})$ method one obtains the QP energies from the linearized QP equation

$$
\varepsilon_{n}^{\mathrm{QP}}=\varepsilon_{n}^{\mathrm{LDA}}+Z_{n}\left\langle\psi_{n}^{\mathrm{LDA}}\left|\Sigma_{G W}\left(\varepsilon_{n}^{\mathrm{LDA}}\right)-v_{\mathrm{xc}}\right| \psi_{n}^{\mathrm{LDA}}\right\rangle,
$$

where $\psi_{n}^{\mathrm{LDA}}$ and $\varepsilon_{n}^{\mathrm{LDA}}$ are LDA eigenstates and eigenvalues, and

$$
Z_{n}=\left[1-\left.\frac{\partial\left\langle\psi_{n}^{\mathrm{LDA}}\left|\Sigma_{G W}(\varepsilon)\right| \psi_{n}^{\mathrm{LDA}}\right\rangle}{\partial \varepsilon}\right|_{\varepsilon_{n}^{\mathrm{LDA}}}\right]^{-1}
$$

The self-energy, $\Sigma_{G W}$, is evaluated non-selfconsistently from the single-particle Green function, i.e., $\Sigma_{G W}=i G_{0} W_{0}$, with $G_{0}(z)=\left(z-H^{\mathrm{LDA}}\right)^{-1}$. It is customary to use the random phase approximation for the screened interaction, i.e., $W_{0}=V(1-V P)^{-1}$ with $P=-i G_{0} G_{0}$.

We have performed the $G_{0} W_{0}$ calculations with the YAMBO $\operatorname{code}^{25}$ using the LDA wave functions and eigenvalues from the PWSCF calculations as input. The plasmon pole approximation has been applied with a frequency of 1 hartree (the HOMO and LUMO energies of benzene change by less than $0.05 \mathrm{eV}$ when the plasmon frequency is varied between 0.5 and 2.0 hartrees). In the calculation of the self-energy we included a minimum of 200 empty states. We have checked that calculations are converged with respect to slab thickness, lateral supercell size, $k$-point mesh, all energy cutoffs and that we reproduce the results previously reported in Ref. 13 for benzene on graphite at $z=3.25 \AA$.

\section{B. Model $G W$ calculations}

In addition to the first-principles $G_{0} W_{0}$ calculations, we have performed (self-consistent) $G W$ calculations for two lattice models representing a metal-molecule and semiconductor-molecule interface, respectively. The model Hamiltonians contain three terms

$$
\hat{H}=\hat{H}_{\mathrm{sol}}+\hat{H}_{\mathrm{mol}}+\hat{U},
$$

describing the solid (metal or semiconductor), the molecule, and their mutual interaction, see Fig. 2. A metallic substrate is modeled by a semi-infinite tight-binding (TB) chain (we suppress the spin for notational simplicity), 

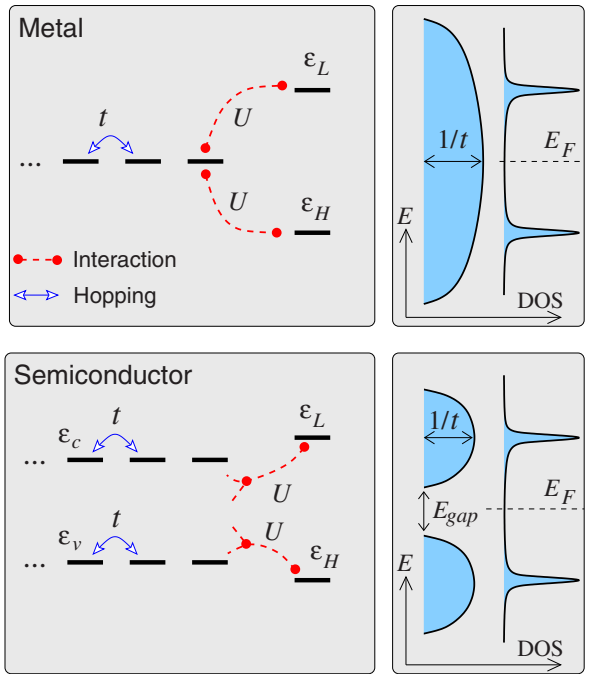

FIG. 2. (Color online) The lattice models representing a metalmolecule and semiconductor-molecule interface, respectively. We consider the weak coupling limit where no hybridization between the molecule and surface states occur. Thus the only interaction between the solid and molecule is via the nonlocal Coulomb interaction $U$.

$$
\hat{H}_{\mathrm{met}}=\sum_{i=-\infty}^{0} t\left(c_{i}^{\dagger} c_{i-1}+c_{i-1}^{\dagger} c_{i}\right) .
$$

A semiconducting substrate is modeled by

$$
\hat{H}_{\mathrm{sc}}=\sum_{\alpha=c, v} \sum_{i=-\infty}^{0} \varepsilon_{\alpha} \hat{n}_{\alpha i}+t\left(c_{\alpha i}^{\dagger} c_{\alpha i-1}+c_{\alpha i-1}^{\dagger} c_{\alpha i}\right),
$$

where $\alpha=c, v$ refers to conduction and valence bands, respectively.

The molecule is represented by its HOMO and LUMO levels, i.e.,

$$
\hat{H}_{\mathrm{mol}}=\xi_{H} \hat{n}_{H}+\xi_{L} \hat{n}_{L}
$$

where e.g., $\hat{n}_{H}=c_{H \uparrow}^{\dagger} c_{H \uparrow}+c_{H \downarrow}^{\dagger} c_{H \downarrow}$, is the number operator of the HOMO level.

Finally, the interaction between the molecule and the terminal site(s) of the substrate TB chain(s) is described by

$$
\hat{U}= \begin{cases}U \hat{n}_{0} \hat{N}_{\mathrm{mol}} & \text { for metals } \\ U \sum_{\sigma}\left(c_{c 0, \sigma}^{\dagger} c_{v 0, \sigma}+c_{v 0, \sigma}^{\dagger} c_{c 0, \sigma}\right) \hat{N}_{\mathrm{mol}} & \text { for semicond }\end{cases}
$$

where $\hat{N}_{\text {mol }}=\hat{n}_{H}+\hat{n}_{L}$ is the number operator of the molecule. Note that since polarization of a semiconductor occurs via transitions between valence and conduction bands, only the interaction terms of the form given above contribute to the image charge effect (this will become clear in Sec. IV A).

We set $E_{F}=0$ corresponding to a half filled band for the metal. We choose $\xi_{H}$ and $\xi_{L}$ so that the molecule contains exactly two electrons ( $E_{F}$ in the middle of the HOMOLUMO gap). We consider the limit of zero hybridization between the solid and molecule so that interaction between the solid and molecule occurs only via the nonlocal $\hat{U}$. The

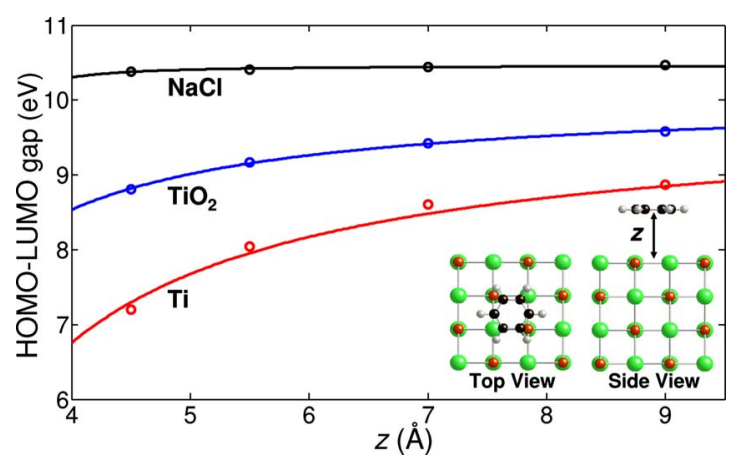

FIG. 3. (Color online) Calculated $G_{0} W_{0}$ energy gap of benzene on $\mathrm{NaCl}, \mathrm{TiO}_{2}$, and $\mathrm{Ti}$ surfaces (circles) as a function of the distance to the surface, and the best fit to the classical model Eq. (8) (full lines).

model neglects interactions within the TB chain and between the molecule and interior TB sites $(i<0)$. These approximations are, however, not expected to influence the image charge physics described by the model in any qualitative way.

We obtain the Green function of the molecule from

$$
G(z)=1 /\left(z-H_{\mathrm{mol}}-\Sigma_{G W}[G](\varepsilon)\right)
$$

where the Hartree potential due to $\hat{U}$ has been absorbed in $H_{\text {mol }}$. The $G W$ self-energy is calculated fully self-consistently using a recently developed $G W$ scheme for quantum transport. $^{26}$ The renormalized molecular QP levels are obtained as peaks in the spectral function $A_{\nu}(\varepsilon)=-(1 / \pi) \operatorname{Im} G_{\nu \nu}^{r}(\varepsilon)$.

\section{CLASSICAL THEORY}

In this section we investigate to what extent the $G_{0} W_{0}$ results of Fig. 1 can be described by a classical image charge model. The electrostatic energy of a point charge, $q$, located in vacuum at position $(0,0, z)$ above a polarizable medium filling the half-space $z<z_{0}$, is given by (in a.u.)

$$
V=\frac{q q^{\prime}}{4\left(z-z_{0}\right)} .
$$

The size of the image charge is $q^{\prime}=q(1-\epsilon) /(1+\epsilon)$, where $\epsilon$ is the relative dielectric constant of the medium. ${ }^{27}$ In 1973 Lang and Kohn showed that the energy of a classical point charge above a quantum jellium surface follows Eq. (8) with $q^{\prime}=-q$ (corresponding to $\epsilon=\infty$ as expected for a perfect metal), with the image plane, $z_{0}$, lying $0.5-0.9 \AA$ outside the surface depending on the electron density. ${ }^{28}$ More recently, ab initio $G_{0} W_{0}$ calculations have found the same asymptotic form for the potential felt by an electron outside a metallic surface. ${ }^{27,29,30}$ From this it seems reasonable to conclude that the asymptotic position of the electronic levels of a molecule outside a surface would also follow the image potential of Eq. (8). This is, however, only true for the unoccupied levels whereas the occupied levels experience a shift in the opposite direction, i.e., the shift is upward in energy as the molecule approaches the surface. This is because the occupied 
TABLE I. Position of the effective image plane, $z_{0}$, and dielectric constant, $\epsilon_{\text {model }}$, obtained by fitting the $z$ dependence of the HOMO-LUMO gap to Eq. (8). Last row shows the experimental optical dielectric constant of the bulk. The two values for the nonisotropic $\mathrm{TiO}_{2}$ refers to longitudinal and transverse polarization directions. Experimental data taken from Ref. 33.

\begin{tabular}{lccc}
\hline \hline & $z_{0}(\AA)$ & $\epsilon_{\text {model }}$ & $\epsilon_{\infty}^{\text {exp,bulk }}$ \\
\hline $\mathrm{NaCl}(001)$ & 1.70 & 1.15 & 2.30 \\
$\mathrm{MgO}(001)$ & 1.20 & 2.63 & 2.95 \\
$\mathrm{CaO}(001)$ & 2.69 & 1.56 & 3.30 \\
$\mathrm{BaO}(001)$ & 2.74 & 1.77 & 3.83 \\
$\mathrm{TiO}(001)$ & 1.79 & 2.76 & $8.43 / 6.84$ \\
$\mathrm{Al}(111)$ & 0.55 & $\infty$ & $\infty$ \\
$\mathrm{Pt}(111)$ & 0.60 & $\infty$ & $\infty$ \\
$\mathrm{Rh}(111)$ & 1.28 & $\infty$ & $\infty$ \\
$\mathrm{Ti}(001)$ & 1.66 & $\infty$ & $\infty$ \\
$\mathrm{Li}(001)$ & 1.72 & $\infty$ & $\infty .83$ \\
$\mathrm{BaO}(111)$ & 2.01 & $\infty$ & \\
\hline \hline
\end{tabular}

levels represent the negative of the energy cost of removing an electron from the molecule. Similarly it has been found that the image potential leads to band gap narrowing at semiconductor-metal interfaces. ${ }^{31,32}$

To test whether the gap reductions obtained in the $G_{0} W_{0}$ calculations can be described by the classical image charge model we have fitted Eq. (8) to the calculated HOMOLUMO gap for $z=4.5,5.5,7.0,9.0, \infty \AA$ A In Fig. 3 we show the result of the fit for three systems (the fit is equally good for the other systems). The best-fit values for the effective image plane $z_{0}$ and the dielectric constant $\epsilon_{\text {model }}$ are given in Table I.

As can be seen $\epsilon_{\text {model }}$ is generally smaller than the experimental optical dielectric constant of the bulk, $\epsilon_{\infty}^{\text {exp,bulk }}$. This is expected since the latter gives the long-range response of the bulk while $\epsilon_{\text {model }}$ probes the local response at the surface. Part of the discrepancy between $\epsilon_{\infty}^{\mathrm{exp}}$ and $\epsilon_{\text {model }}$ is clearly due to geometric effects. By taking the surface geometry into account, as done in Ref. 18, better estimates of $\epsilon_{\text {model }}$ can be produced from $\epsilon_{\infty}^{\exp }$. On the other hand, electronic effects due to the local atomic structure of the surface cannot be captured by a classical model. For example, the $\mathrm{BaO}(111)$ surface is metallic due to surface states, and thus $\epsilon_{\text {model }} \approx \infty$ while $\epsilon_{\infty}^{\exp }=3.83$. Similarly, impurities, defects, and surface roughness are expected to influence the local dielectric properties of the surface.

According to the classical image charge model all the molecular levels should experience the same shift (the sign of the shift being different for occupied and unoccupied levels). However, we have found that the best-fit values for $z_{0}$ and $\epsilon_{\text {model }}$ obtained by fitting the HOMO and LUMO levels separately, are in general different-most notably for the metallic surfaces. This observation, which is discussed in more detail in Sec. IV C, shows that the shape of molecular orbital also influences the size of the polarization-induced shift.

\section{MICROSCOPIC THEORY}

In this section we first consider the $G W$ self-energy for a molecule interacting with a surface to second-order in the electron-electron interaction. This leads to a simple microscopic model for image charge renormalization which relates the shift of molecular levels to the electronic structure of the surface, and explains general trends of the first-principles and model $G W$ calculations. In the last section we consider the HOMO and LUMO levels separately and explain how error cancellations in semilocal exchange-correlation functionals can explain the surprisingly good agreement found between LDA eigenvalues and $G W \mathrm{QP}$ energies for the occupied levels of benzene on metallic surfaces.

\section{A. Second-order expansion}

In quantum many-body theory, the effect of substrate polarization on the energy levels of a molecule enters the Green function via a self-energy operator. In general, the $G_{0} W_{0}$ selfenergy can be written symbolically as

$$
\Sigma=\sum_{n=1} \Sigma^{(n)}=\sum_{n=1} i G_{0} V(P V)^{n-1},
$$

where $G_{0}$ is the Green function of the noninteracting (KohnSham) Hamiltonian, and $P=-i G_{0} G_{0}$ is the polarization bubble. The first-order term, $\Sigma^{(1)}$, is simply the static exchange potential while the remaining terms account for correlations and dynamic screening. In the following we consider the second-order term, $\Sigma^{(2)}=i G_{0} V P V$ explicitly. This corresponds to approximating the response of the substrate by its noninteracting response, $P$.

For sufficiently large surface-molecule separations $(z \geq 3.5 \AA)$ we can neglect hybridization effects, and the noninteracting eigenstates of the combined system can be taken as the eigenstates of the isolated molecule and surface. We denote these eigenstates by $\left\{\psi_{a}\right\}$ (" $a$ " for adsorbate) and $\left\{\psi_{k}\right\}$, respectively. To see how a given electronic level, $\varepsilon_{a}$, is renormalized by polarization processes in the substrate we consider the (time-ordered) matrix element $\Sigma_{a a}^{(2)}(\omega)$ $=\left\langle\psi_{a}\left|\Sigma^{(2)}(\omega)\right| \psi_{a}\right\rangle$, given by

$$
\Sigma_{a a}^{(2)}=\sum_{k}^{\text {occ empty }} \sum_{k^{\prime}} \int i G_{0, a a}\left(\omega^{\prime}\right) V_{a a, k k^{\prime}} P_{k k^{\prime}}\left(\omega^{\prime}-\omega\right) V_{k^{\prime} k, a a} d \omega^{\prime} .
$$

The Feynman diagram corresponding to $\Sigma_{a a}^{(2)}$ is shown in Fig. 4(a). The polarization and Coulomb matrices are given by

$$
\begin{aligned}
P_{k k^{\prime}}(\omega) & =\frac{1}{\omega-\omega_{k k^{\prime}}+i \eta}-\frac{1}{\omega+\omega_{k k^{\prime}}-i \eta} \\
V_{k k^{\prime}, a a} & =\iint \frac{\psi_{k}^{*}(\mathbf{r}) \psi_{k^{\prime}}(\mathbf{r})\left|\psi_{a}\left(\mathbf{r}^{\prime}\right)\right|^{2}}{\left|\mathbf{r}-\mathbf{r}^{\prime}\right|} d \mathbf{r} d \mathbf{r}^{\prime}
\end{aligned}
$$

where $\eta$ is a positive infinitesimal and $\omega_{k k^{\prime}}=\varepsilon_{k^{\prime}}-\varepsilon_{k} \geq 0$. Using that $G_{0, a a}(\omega)=1 /\left(\omega-\varepsilon_{a}+\operatorname{sgn}\left(\varepsilon_{a}-E_{F}\right) i \eta\right),{ }^{11}$ Eq. (10) reduces to 
(a)

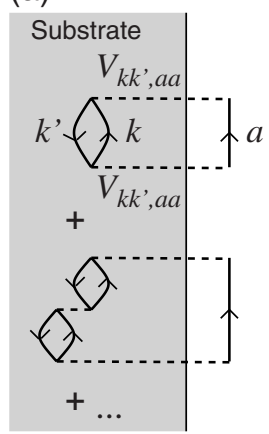

(b)

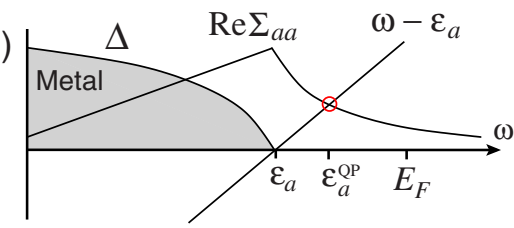

(c)

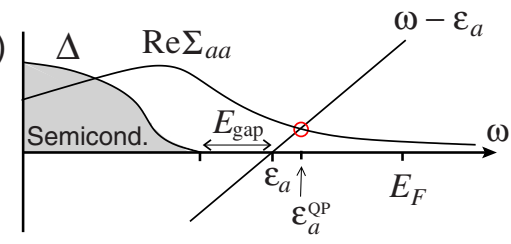

FIG. 4. (Color online) (a) Feynman diagrams representing dynamic polarization of the substrate induced by an electron propagating in the molecule. (b) and (c): generic shapes of the imaginary and real parts of the self-energy of Eq. (15) for an occupied molecular level $|a\rangle$ interacting with a metallic and semiconducting substrate assuming $V_{k k^{\prime}, a a}$ to be energy independent.

$$
\Sigma_{a a}^{(2)}(\omega)=\frac{1}{\pi} \int \frac{\Delta\left(\omega^{\prime}\right)}{\omega-\omega^{\prime}+\operatorname{sgn}\left(E_{F}-\varepsilon_{a}\right) i \eta} d \omega^{\prime}
$$

where we have defined the interaction strength,

$$
\Delta=\pi \sum_{k}^{\text {occ empty }} \sum_{k^{\prime}}\left|V_{k k^{\prime}, a a}\right|^{2} \delta\left(\omega_{k k^{\prime}}-\operatorname{sgn}\left(\varepsilon_{a}-E_{F}\right)\left(\omega-\varepsilon_{a}\right)\right) .
$$

Note that $\Delta$ is simply the joint density of states (JDOS) of the substrate, shifted by $\varepsilon_{a}$, and weighted by the Coulomb matrix elements. The physically relevant retarded self-energy is readily obtained from Eq. (13)

$$
\Sigma_{a a}^{(2), r}=\frac{\mathcal{P}}{\pi} \int \frac{\Delta\left(\omega^{\prime}\right)}{\omega^{\prime}-\omega} d \omega^{\prime}-i \Delta(\omega) .
$$

where $\mathcal{P}$ denotes the Cauchy principal value. Now, the renormalized QP energy can be obtained from the equation (neglecting off-diagonal terms)

$$
\varepsilon_{a}^{\mathrm{QP}}-\varepsilon_{a}-\operatorname{Re}\left\langle\psi_{a}\left|\Sigma^{(2), r}\left(\varepsilon_{a}^{\mathrm{QP}}\right)\right| \psi_{a}\right\rangle=0
$$

A graphical solution to the QP equation is illustrated in Figs. 4(b) and 4(c) for the case of an occupied molecular level $\varepsilon_{a}<E_{F}$ interacting with a metal or semiconductor surface, respectively.

From Eq. (14) it follows that the image charge effect does not broaden the molecular level because $\operatorname{Im} \Sigma^{(2)}\left(\varepsilon_{a}\right)=0$. We also note that the level shift is independent of the absolute value $\left|\varepsilon_{a}-E_{F}\right|$, and that the effect of changing the sign of $\varepsilon_{a}-E_{F}$ is to change the sign of the level shift. These properties are all in line with the classical theory.

In the limit where $V_{k k^{\prime}, a a}$ varies little with $k$ and $k^{\prime}, \Delta$ is simply proportional to the shifted JDOS [the "generic" cases illustrated in Figs. 4(b) and 4(c)]. In this case the level shift is simply determined by the form of the JDOS. For a metal, the JDOS raises linearly at $\omega=0$ with a slope given by the metal's DOS at $E_{F}$. This suggests that the level shift should increase with the substrate DOS at the Fermi level. For a

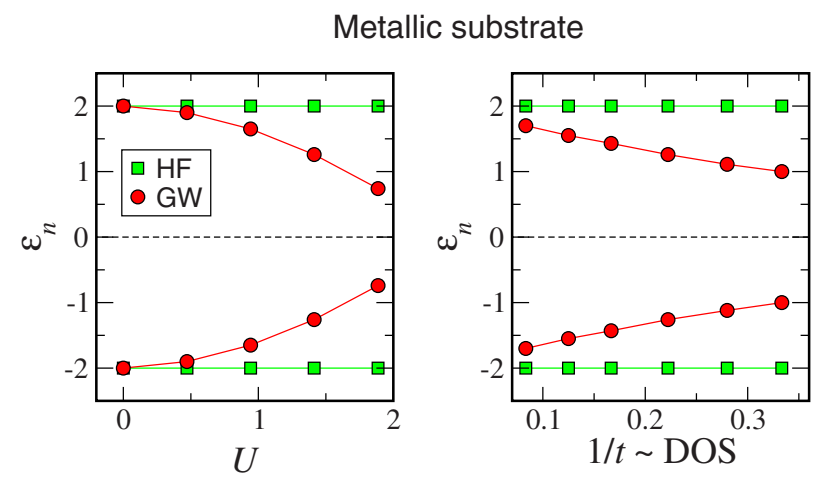

Semiconducting substrate
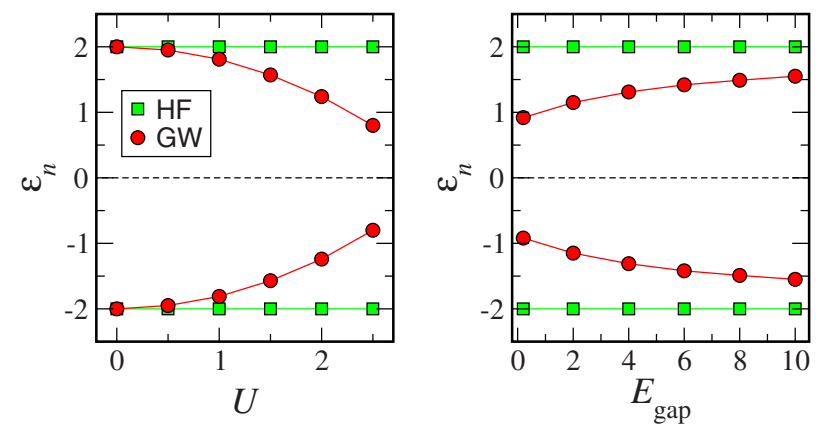

FIG. 5. (Color online) HOMO and LUMO positions obtained from the simple lattice models for a metallic substrate (upper panel) and semiconducting substrate (lower panel). In all plots we vary one parameter while keeping the remaining parameters fixed. ${ }^{34}$

semiconductor, the JDOS raises smoothly at $\omega=E_{\text {gap }}$, suggesting that the level shift should decrease with $E_{\text {gap }}$. In the following section we investigate these relations for the model and first-principles calculations. We mention that the second-order approximation discussed above may not always provide a good description of the full $G W$ self-energy. However, as we will show in the next section, it explains qualitatively the trends in $G_{0} W_{0}$ calculations.

\section{B. Dependence of level shift on surface electronic structure}

In Fig. 5 we show the HOMO and LUMO levels of the lattice models calculated with the $\mathrm{HF}$ and $G W$ approximations. In all plots we vary one parameter of the model while keeping the remaining parameters fixed. ${ }^{34}$

The upper panels refer to a metallic substrate and show the dependence on the levels on the interaction strength $U$ and the intrachain hopping parameter $t$. Note that the latter is inversely proportional to the projected density of states (DOS) of the terminal site evaluated at $E_{F}$. The lower panels refer to a semiconducting substrate and show the dependence of the levels on $U$ and the substrate gap, $E_{\text {gap }}$. The HF eigenvalues are clearly independent of the nonlocal interaction between the molecule and substrate. This can be understood from Koopmans' theorem which states that the HF eigenvalues do not include the electronic relaxations of the substrate induced by the extra electron/hole in the molecule. In contrast the $G W$ levels vary in the way predicted by the simple model discussed in the previous section: The polarization- 

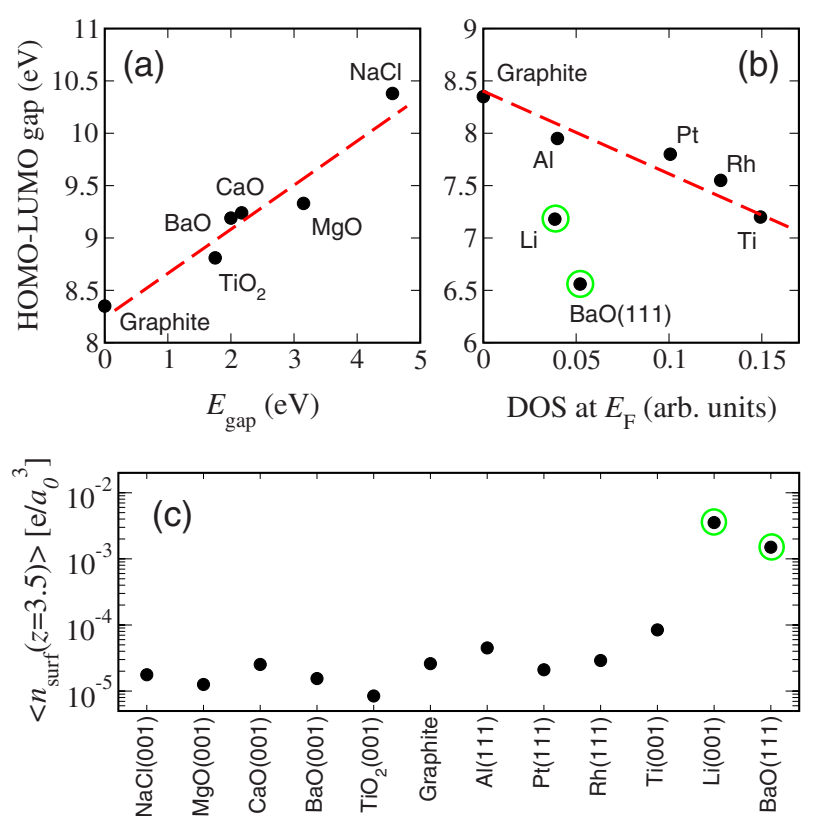

FIG. 6. (Color online) Calculated HOMO-LUMO gap of benzene at $z=4.5 \AA$ (same numbers as in Fig. 1) plotted as function of the LDA substrate band gap for the semiconductors (a) and the total DOS per volume evaluated at the Fermi level for the metals (b). Dashed lines have been added to guide the eye. (c) Average electron density in a plane lying $z=3.5 \AA$ above the clean surfaces.

induced reduction of the HOMO-LUMO gap is stronger for larger $U$ as well as for larger substrate DOS at $E_{F}$ for the metals and smaller substrate band gap for the semiconductors. A more detailed discussion of level renormalization based on the lattice model for metallic substrates, including the case of strong metal-molecule hybridization, can be found in Ref. 15.

In Figs. 6(a) and 6(b) we plot the $G_{0} W_{0}$ gaps from Fig. 1 versus the LDA band gap and DOS at $E_{F}$ for the semiconducting and metallic substrates, respectively. For the semiconductors the reduction of the HOMO-LUMO gap clearly correlates with $E_{\text {gap. }}$. This indicates that the interaction strength, i.e., the matrix elements $V_{k k^{\prime}, a a}$ of Eq. (14), do not differ too much from one surface to another. For the metals, the HOMO-LUMO gap seems to scale with the metal's DOS at $E_{F}$. However, we note that $\mathrm{Li}(001)$ and $\mathrm{BaO}(111)$ deviate from the general trend followed by the other metals. This can be explained by the larger extend of the metallic wave functions of these systems into the vacuum region, which in turn leads to larger $V_{k k^{\prime}, a a}$ matrix elements. Indeed, Fig. 6(c) shows the average electron density evaluated in a plane lying $z=3.5 \AA$ above the surface in the absence of the benzene molecule. The density outside the $\mathrm{Li}(001)$ and $\mathrm{BaO}(111)$ surfaces is significantly larger than for the other surfaces which on the other hand have quite similar densities.

\section{DFT eigenvalues and error cancellation}

In Fig. 7 we plot the energies of the HOMO and LUMO levels of benzene at $z=4.5 \AA$. For each surface, we have shifted the LDA, PBE0, and $G_{0} W_{0}$ levels by the same

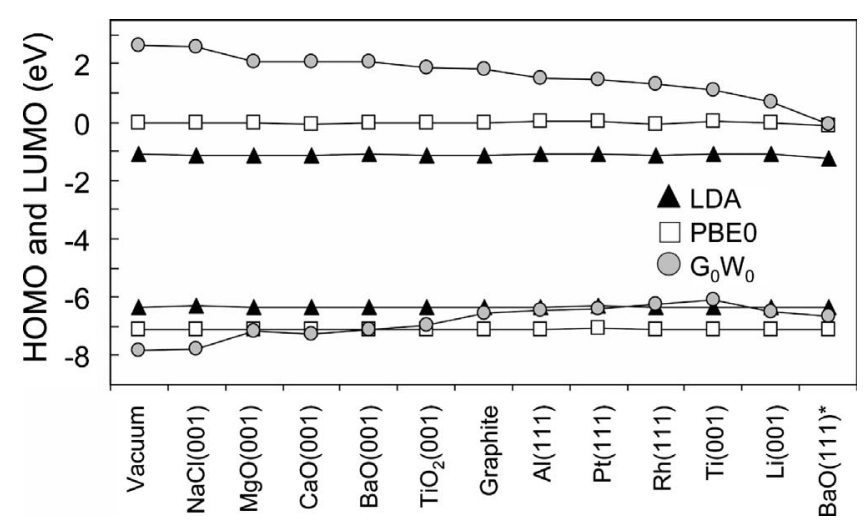

FIG. 7. LDA, PBE0, and $G_{0} W_{0}$ energies for the HOMO and LUMO levels of benzene at $z=4.5 \AA$ above the surfaces. The very good agreement between LDA and $G_{0} W_{0}$ energies for the HOMO level at the metallic surfaces is due to error cancellation in the LDA approximation.

amount so that the LDA HOMO is aligned with the HOMO in the gas phase. We note that the effect of substrate polarization is very similar for the $G_{0} W_{0}$ HOMO and LUMO levels which are shifted up and down, respectively, by almost the same amount. This is indeed expected from the classical image charge model. Significant deviations from this trend are, however, seen for $\mathrm{Li}(001)$ and $\mathrm{BaO}(111)$. We ascribe this to the more extended nature of the metallic states on these surfaces which reduce the validity of the point charge approximation and can introduce differences between the $V_{k k^{\prime}, H H}$ and $V_{k k^{\prime}, L L}$ matrix elements.

Overall, the LDA and PBE0 eigenvalues for the HOMO are in better agreement with the $G_{0} W_{0} \mathrm{QP}$ energies than is the case for the LUMO. Moreover there is a general trend that the LDA eigenvalues come closer to the $G_{0} W_{0}$ energies as we move from the insulating to the metallic surfaces. In fact, the LDA HOMO level is almost on top of the $G_{0} W_{0}$ level on the metallic surfaces. This trend is clearly a result of significant error cancellation in the LDA. Indeed, it is well known that semilocal exchange-correlation functionals overestimate (underestimate) occupied (empty) molecular levels due to self-interaction effects. At the metallic surfaces this error is compensated by the missing image charge correction. PBE0 gives better estimates for the free molecule where it opens up the LDA HOMO-LUMO gap due to partial removal of self-interaction errors. In this case, the cancellation between the missing image charge effect and the remaining self-interaction error results in very good agreement between PBE0 and $G_{0} W_{0}$ for the HOMO level on the semiconducting surfaces.

The cancellation between self-interaction errors and missing polarization effects will always be present in hybrid- and semilocal approximations. However, the relative size of the two contributions will in general depend on the shape of the molecule, its orientation with respect to the surface, the molecule-surface distance, and the type of substrate.

\section{CONCLUSIONS}

We have presented $G_{0} W_{0}$ calculations for a benzene molecule physisorbed on different metallic and semiconducting 
surfaces. Upon physisorption the molecule's HOMO-LUMO gap is reduced from its gas phase value due to dynamic polarization of the substrate. It was shown that a classical image charge model captures the qualitative features of the effect while the magnitude of the level shift is sensitive to the detailed atomic structure of the surface. In particular the presence of metallic midgap state at the surface of a semiconductor can have a large influence on the local response of the surface. Both local and hybrid exchange-correlation potentials fail to account for the polarization effects yielding Kohn-Sham eigenvalues of physisorbed benzene which are independent of the substrate. Nevertheless we found that a cancellation between self-interaction errors and missing image charge effects in the LDA leads to a very good agreement between LDA and $G_{0} W_{0}$ energies for the occupied states of benzene on metallic surfaces. Similar conclusions were reached for the PBE0 energies on semiconducting substrates. Finally, we have derived a simple second-order approximation to the $G W$ self-energy which expresses the polarization-induced shift of a molecular level in terms of the substrate's joint density of states weighted by Coulomb in- teraction matrix elements. This model was used to explain general trends in the first-principles results, namely the scaling of the benzene's HOMO-LUMO gap with the substrate density of states at $E_{F}$ (for metals) and the substrate band gap (for semiconductors).

Our results clearly demonstrate the importance of nonlocal correlations for the electronic levels at solid-molecule interfaces. We expect this to have important implications for the theoretical modeling of electron transport in organic and single-molecule devices.

\section{ACKNOWLEDGMENTS}

K.S.T. and C.R. acknowledge support from the Danish Center for Scientific Computing. The Center for Atomicscale Materials Design (CAMD) is sponsored by the Lundbeck Foundation. A.R. and J.M.G.L. acknowledge funding by the Spanish MEC (Grant No. FIS2007-65702-C02-01), "Grupos Consolidados UPV/EHU del Gobierno Vasco" (Grant No. IT-319-07), e-I3 ETSF project (Contract No. 211956) and "Red Española de Supercomputación."
${ }^{1}$ J. K. Nørskov, T. Bligaard, J. Rossmeisl, and C. H. Christensen, Nat. Chem. 1, 37 (2009).

${ }^{2}$ K. Moth-Poulsen and T. Bjornholm, Nat. Nanotechnol. 4, 551 (2009).

${ }^{3}$ A. Nitzan and M. A. Ratner, Science 300, 1384 (2003).

${ }^{4}$ W. A. Hofer, A. S. Foster, and A. L. Shluger, Rev. Mod. Phys. 75, 1287 (2003).

${ }^{5}$ F. Flores, J. Ortega, and H. Vazquez, Phys. Chem. Chem. Phys. 11, 8658 (2009).

${ }^{6}$ P. D. Johnson and S. L. Hulbert, Phys. Rev. B 35, 9427 (1987).

${ }^{7}$ S. Kubatkin,Andrey Danilov, Mattias Hjort, Jérôme Cornil, Jean-Luc Brédas, Nicolai Stuhr-Hansen, Per Hedegård, and Thomas Bjørnholm, Nature (London) 425, 698 (2003).

${ }^{8}$ R. Hesper, L. H. Tjeng, and G. A. Sawatzky, Europhys. Lett. 40, 177 (1997).

${ }^{9}$ J. Repp, G. Meyer, S. M. Stojković, A. Gourdon, and C. Joachim, Phys. Rev. Lett. 94, 026803 (2005).

${ }^{10}$ A. Kahn, N. Koch, and W. Gao, J. Polym. Sci., B, Polym. Phys. 41, 2529 (2003).

${ }^{11}$ F. Aryasetiawan and O. Gunnarsson, Rep. Prog. Phys. 61, 237 (1998).

${ }^{12}$ G. Onida, L. Reining, and A. Rubio, Rev. Mod. Phys. 74, 601 (2002).

${ }^{13}$ J. B. Neaton, Mark S. Hybertsen, and Steven G. Louie, Phys. Rev. Lett. 97, 216405 (2006).

${ }^{14}$ C. Freysoldt, P. Rinke, and M. Scheffler, Phys. Rev. Lett. 103, 056803 (2009).

${ }^{15}$ K. S. Thygesen and A. Rubio, Phys. Rev. Lett. 102, 046802 (2009).

${ }^{16}$ S. Y. Quek, Latha Venkataraman, Hyoung Joon Choi, Steven G.
Louie, Mark S. Hybertsen, and J. B. Neaton, Nano Lett. 7, 3477 (2007).

${ }^{17}$ D. J. Mowbray, G. Jones, and K. S. Thygesen, J. Chem. Phys. 128, 111103 (2008).

${ }^{18}$ K. Kaasbjerg and K. Flensberg, Nano Lett. 8, 3809 (2008).

${ }^{19}$ R. Stadler, V. Geskin, and J. Cornil, Phys. Rev. B 79, 113408 (2009).

${ }^{20} \mathrm{~S}$. Baroni et al., QUANTUM-ESPRESSO package, 2005 (http:// www.quantum-espresso.org/).

${ }^{21}$ M. Fuchs and M. Scheffler, Comput. Phys. Commun. 119, 67 (1999).

${ }^{22}$ J. P. Perdew and Y. Wang, Phys. Rev. B 45, 13244 (1992).

${ }^{23}$ M. Ernzerhof and G. Scuseria, J. Chem. Phys. 110, 5029 (1999).

${ }^{24}$ C. Adamo and V. Barone, J. Chem. Phys. 110, 6158 (1999).

${ }^{25}$ A. Marini, C. Hogan, M. Grüning, and D. Varsano, Comput. Phys. Commun. 180, 1392 (2009).

${ }^{26}$ K. S. Thygesen and A. Rubio, Phys. Rev. B 77, 115333 (2008).

${ }^{27}$ M. Rohlfing, N.-P. Wang, P. Krüger, and J. Pollmann, Phys. Rev. Lett. 91, 256802 (2003).

${ }^{28}$ N. Lang and W. Kohn, Phys. Rev. B 7, 3541 (1973).

${ }^{29}$ A. G. Eguiluz, M. Heinrichsmeier, A. Fleszar, and W. Hanke, Phys. Rev. Lett. 68, 1359 (1992).

${ }^{30}$ I. D. White, R. W. Godby, M. M. Rieger, and R. J. Needs, Phys. Rev. Lett. 80, 4265 (1998).

${ }^{31}$ J. C. Inkson, J. Phys. C 6, 1350 (1973).

${ }^{32}$ J. P. A. Charlesworth, R. W. Godby, and R. J. Needs, Phys. Rev. Lett. 70, 1685 (1993).

${ }^{33}$ www.oxmat.co.uk/crysdata/homepage.htm.

${ }^{34}$ For the metals: $t=5, U=2, \xi_{H / L}= \pm 2-U$. For the semiconductor: $t=2, U=2, \xi_{H / L}= \pm 2$, and $\varepsilon_{c / v}= \pm 5.5$. 